\title{
Kinematic Reducibility of Multiple Model Systems
}

\author{
T.D. Murphey and J.W. Burdick \\ Engineering and Applied Science \\ California Institute of Technology \\ Mail Code 104-44, Pasadena, CA 91125 USA \\ \{murphey, jwb\} arobotics.caltech. edu
}

\begin{abstract}
This paper considers the relationship between second order multiple model systems and first order multiple model systems. Such a relationship is important to, among other things, studying path planning for mechanical control systems. This is largely due to the fact that the computational complexity of a path planning problem rapidly increases with the dimension of the state space, implying that being able to reduce a path planning problem from $T Q$ to $Q$ can be helpful. Not surprisingly, the necessary and sufficient condition for such a reduction is that each model constituting a multiple model control system be reducible. We present an extensive example in order to illustrate how these results can provide insight into the control of some specific physical systems.
\end{abstract}

\section{INTRODUCTION}

Many mechanical systems, though intrinsically second order in a mechanical framework, can be described well by first order equations of motion. Classical examples of such systems include the well studied kinematic car, the hopping robot, and the forward kinematics of a robotic arm. The benefits of this simplification are numerous: the dimension of the configuration space drops by half as the configuration space goes from $T Q$ to $Q$ and inputs go from being forces to being velocities, something which is often more easily realized physically. One of the main goals of this paper is to show that this type of reduction can also be achieved for what we term multiple model systems, that is, systems which have several possible models describing the state evolution.

Reduction theory has its roots in work by [15], [4], [1], [16] among many other works. Here, various parts of the configuration manifold are taken out of the representation of the dynamics, and then reconstructed later. These often correspond to symmetries in the motion of the control system. In the case we discuss here, the idea of symmetry will not be so important as that of partial integrability. The main concept is that in some cases one can integrate a system with force inputs to a system with velocity inputs. This is useful, particularly in implementation where it is often more convenient to have velocity inputs (which are easily controlled by low level motor controllers) than to have torque inputs. These ideas have been used in work by [6], [7], [12]. Reductions can have a large impact on both numerical integration and path planning for mechanical systems such as robots working in a complicated ambient space.

This paper is organized as follows. We first go over some basic definitions and concepts in Section II. We describe kinematic reducibility for smooth systems in Section III.
Then we show that this can be easily incorporated into the nonsmooth setting in Section IV. Section V analyzes a very simple vehicle example and then discusses a more complex example similar to the Mars rover. The example of the Mars rover is particularly interesting because it represents what our primary motivation - the Mars rover is an incredibly slowly moving mechanical system and we therefore expect it to have first order characteristics. Section VI gives some final remarks on the relationship between kinematic reducibility and the power dissipation method for finding kinematic equations of motion for overconstrained systems, and how this relationship can be used.

\section{Multiple Model Systems}

We are considering the issue of kinematic reducibility for systems of the following form:

Definition 2.1: A control system $\Sigma$ is said to be a multiple model (MM) system if it can be expressed in the form

$$
\Sigma: \quad \dot{q}=f_{0}+f_{1}(q) u_{1}+f_{2}(q) u_{2}+\cdots+f_{m}(q) u_{m}
$$

where for any $q$ and $t, f_{i} \in\left\{g_{\alpha_{i}} \mid \alpha_{i} \in I_{i}\right\}$, with $I_{i}$ a finite index set, $g_{\alpha_{i}}$ analytic in $(q, t)$ for all $\alpha_{i}$, and the controls $u_{i} \in U \subset \mathbb{R}$ are piecewise constant and $U$ is compact. Moreover, letting $\sigma_{i}$ denote the "switching signals" associated with $f_{i}$ (which will be referred to as "MM maps"),

$$
\begin{aligned}
\sigma_{i}: Q \times \mathbb{R} & \longrightarrow \mathbb{N} \\
(q, t) & \longrightarrow \alpha_{i}
\end{aligned}
$$

then we assume the $\sigma_{i}$ are measurable in $(q, t)$.

A multiple model system is an affine nonlinear control system where each control vector field (including the drift term) may "switch" back and forth between different elements of a finite set. The $\sigma_{i}$ which regulate this switching may not be known, so we have no guarantees about the nature of the switching except that it is measurable. It is a simple consequence of measure theory that $f_{i}$ is measurable in $(q, t)$ since $\sigma_{i}$ is measurable and the $g_{\alpha_{i}}$ are analytic. In our case, this switching corresponds to the switching among different contact states (i.e., different sets of slipping contacts) due to variations in contact geometry and surface friction properties. Moreover, so that we can distinguish between the overall control system and the smooth control systems that comprise it, define the following.

Definition 2.2: Let $\Sigma$ be an MM control system. Then we define $\Sigma_{\sigma_{1}, \sigma_{2}, \ldots, \sigma_{n}}$ to be the individual control systems made 
up of

$$
\Sigma_{\sigma_{0}, \sigma_{1}, \ldots, \sigma_{m}}: \quad \dot{q}=g_{\sigma_{0}}+g_{\sigma_{1}} u_{1}+g_{\sigma_{2}} u_{2}+\cdots+g_{\sigma_{m}} u_{m}
$$

We will additionally refer to a system as a multiple model driftless affine (MMDA) system if it is an $\mathrm{MM}$ system with $f_{0}=0$. That is, it is a multiple model system without a drift term.

Definition 2.1 implies the control vector fields may change, or switch, among a finite collection of vector fields, each representing a model, $P$, in a set of models $\mathcal{P}$. Such systems are intimately related to multiple model systems such as studied in [11], [17], [19]. However, we should emphasize that the "switching" is not like the switching phenomena found in [5], [14], [8], [24], or as typically studied in the hybrid control systems literature (e.g., [22], [3]). In these studies, the switching is part of a control strategy to be implemented in the controller. Rather, it is switching induced by environmental factors, such as variations in the contact state between rigid bodies. Systems of this sort are actually quite common in engineering practice, as anything that has intermittent contact or more kinematic constraints than degrees of freedom must experience these "jumps" in dynamics. The goal is to develop systematic methods for analyzing complicated mechanical systems with the type of hybrid structure seen in Definition 2.1. Section V develops a simple example in detail.

\section{III. $(\mathcal{U}, \overline{\mathcal{U}})$ Reducibility: The Smooth CASE}

The notion of $(\mathcal{U}, \overline{\mathcal{U}})$-reducibility formalizes what is meant by kinematic reducibility. It is basically the requirement that all paths on $T Q$ coincide in the right way with paths on $Q$ when they are projected onto $Q$. The definition can be found near the end of this section. Lewis [12] proved that the symmetric product could be used to provide a local test for reducibility, which we will define rigorously in a moment. Here Lewis' result is extended to the case of overconstrained mechanical systems that are modeled as multiple model systems. The main result states that if all of the individual models comprising the multiple model system are $(\mathcal{U}, \overline{\mathcal{U}})$ reducible, then the multiple model system is $(\mathcal{U}, \overline{\mathcal{U}})$ reducible. Section IV gives the theorem statement and proof regarding $(\mathcal{U}, \overline{\mathcal{U}})$-reducibility of multiple model mechanical systems.

The proof methodology will basically be the following. For each model that makes up a multiple model mechanical system, we will have reduced equations that come from [12]. Any map that has its time derivative in the convex hull of all these model equations is a solution to the multiple model mechanical system. These solutions will be approximated with a limit of solutions that are piecewise explicitly known to be $(\mathcal{U}, \overline{\mathcal{U}})$ reducible. Then they will be reduced to first order equations and a result from Filippov [9] will be used to show that the limit of these in the reduced space is also a solution. Then the process is reversed to show that for any solution to the MMDA system there is a solution to the multiple model mechanical system. Before proceeding to the proof, however, we review $(\mathcal{U}, \overline{\mathcal{U}})$-reducibility for smooth systems.

For mechanical systems we will consider inputs $u$ : $[0, T] \rightarrow \mathbb{R}^{m}$ that are essentially bounded and Lebesgue integrable, and for kinematic systems we will consider inputs that are piecewise constant. In Lewis [12], it was assumed that the inputs were absolutely continuous since piecewise continuous inputs imply that one can change the systems velocity instantaneously. With inertia this can only occur given infinite forces. We will keep this assumption. However, in the present work state transitions are being approximated with piecewise continuous signals. This is a common approximation in many areas of physical modeling-for example, the study of impacting bodies often includes this assumption. Therefore, we will only require that absolute continuity hold almost everywhere in our subsequent treatment.

Definition 3.1: $f:[a, b] \rightarrow \mathbb{R}^{m}$ is absolutely continuous if for each $\epsilon>0 \exists \delta>0$ such that for every finite collection $\left\{\left(t_{i}, t_{i}^{\prime}\right)\right\}_{1 \leq i \leq N}$ of non-overlapping intervals in $[a, b]$ with the property that

$$
\sum_{i=1}^{N}\left|t_{i}^{\prime}-t_{i}\right|<\delta \text { we have } \sum_{i=1}^{N}\left\|f\left(t_{i}^{\prime}\right)-f\left(t_{i}\right)\right\|<\epsilon
$$

This implies $D f$ exists almost everywhere. Let us define some of the basic notions from differential geometry, including the covariant derivative, the distribution, and the symmetric product. As in Lewis [12], we restrict our attention to simple mechanical systems whose Lagrangian takes the form $\mathcal{L}=K \cdot E .-V$. Assume that $Q$ is an $n$-dimensional configuration manifold, and $g$ is a riemannian metric on $Q$ defining the kinetic energy. Also, since many of the applications of interest are systems with no potential energy (such as flat terrain problems), let us simplify to the case where $\mathcal{L}=K$.E. (i.e., $V=0$ ).

First, some more definitions are necessary so that the symmetric product can be defined. (We generally follow Lewis' notation here.) Denote by $v_{q}$ elements in the tangent space of $Q$ at $q, T_{q} Q$. Assuming that the potential energy is zero, the system Lagrangian is $\mathcal{L}=\frac{1}{2} g\left(v_{q}, v_{q}\right)$. Next we recall Christoffel symbols so that we can define the covariant derivative and then the symmetric product.

Definition 3.2: The Christoffel symbols for the Levi-Civita connection $\nabla$ are

$$
\Gamma_{j k}^{i}=\frac{1}{2} g^{i l}\left(\frac{\partial g_{j l}}{\partial q^{k}}+\frac{\partial g_{k l}}{\partial q^{j}}-\frac{\partial g_{j k}}{\partial q^{l}}\right)
$$

where the standard convention of implied summation over repeated indices is used unless otherwise stated and upper indices indicate the inverse.

Now we can define the covariant derivative and finally the symmetric product. 
Definition 3.3: In coordinates, the covariant derivative of $Y$ with respect to $X$ is

$$
\nabla_{X} Y=\left(\frac{\partial Y^{i}}{\partial q^{j}} X^{j}+\Gamma_{j k}^{i} X^{j} Y^{k}\right) \frac{\partial}{\partial q^{j}}
$$

Definition 3.4: The symmetric product between two vector fields $X$ and $Y$ is defined to be

$$
\langle X: Y\rangle=\nabla_{X} Y+\nabla_{Y} X
$$

Given a metric $g$ on the manifold $Q$ and inputs $u^{\alpha}$, it is possible to show that the Euler-Lagrange equations can be written in the form:

$$
\nabla_{c^{\prime}(t)} c^{\prime}(t)=u^{a}(t) Y_{a}(c(t))
$$

where $t \rightarrow c(t)$ is a path on $Q$ and $c^{\prime}(t)=\frac{d}{d t} c(t)$. On the other hand, given input velocities $\bar{u}^{\alpha}$, kinematic equations can be written in the form:

$$
\dot{q}(t)=\bar{u}^{\alpha}(t) X_{\alpha}(q(t))
$$

Let $\left\{Y_{1}, \ldots, Y_{m}\right\}$ and $\left\{X_{1}, \ldots, X_{\bar{m}}\right\}$ be two sets of vector fields on $T Q$ for $m, \bar{m} \in \mathbb{N}$. Denote by $D_{d y n}$ the distribution spanned by the vector fields $\left\{Y_{1}, \ldots, Y_{m}\right\}$ and by $D_{k i n}$ the distribution spanned by the vector fields $\left\{X_{1}, \ldots, X_{\bar{m}}\right\}$. Although the formulation is not presented here, we will use the fact that mechanical systems with constraints can be written in the form of Equation (5) [13]. The next definition formalizes the class of admissible solutions to Equation (5) and Equation (6).

Definition 3.5: If we have a control system $\dot{q}=f(q, u)$ on $Q$ and $u$ coming from some space of inputs $U \subseteq \mathbb{R}^{m}$, a $(\mathcal{U}, \mathcal{T})$-solution is a pair $(c, u)$, where $u:[0, T] \rightarrow U$ and $c:[0, T] \rightarrow Q$ satisfy $c^{\prime}(t)=f(c(t), u(t))$. Let

$$
\begin{aligned}
\tau_{Q}: & T Q \\
\left(v_{q}, q\right) & \rightarrow Q
\end{aligned}
$$

be the tangent bundle projection. We now can define what it means for a mechanical system of the form in Equation (5) to be $(\mathcal{U}, \bar{U})$ reducible to Equation (6).

Definition 3.6: Let $\nabla$ be an affine connection on $Q$, and let $\mathcal{U}$ and $\bar{U}$ be two families of control functions. The system in Equation (5) is $(\mathcal{U}, \overline{\mathcal{U}})$-reducible to the system in Equation (6) if the following two conditions hold:

i ) for each $(\mathcal{U}, \mathcal{T})$-solution $(\eta, u)$ of Equation (5) with initial conditions $\eta(0)$ in the distribution $D_{k i n}$, there exists a $(\overrightarrow{\mathcal{U}}, \mathcal{T})$-solution $(c, \bar{u})$ of Equation (6) with the property that $c=\tau_{Q} \circ \eta$;

ii ) for each $(\overline{\mathcal{U}}, \mathcal{T})$-solution $(c, \bar{u})$ of Equation (6), there exists a $(\mathcal{U}, \mathcal{T})$-solution $(\eta, u)$ of Equation (5) with the property that $\eta(t)=c^{\prime}(t)$ for almost every $t \in[0, T]$.

Let $\chi^{\infty}(D)$ denote those $C^{\infty}$ vector fields taking values in the distribution $D$. The following theorem states the local test for Equation (5) to be $(\mathcal{U}, \overline{\mathcal{U}})$ reducible to Equation (6).
Theorem 3.1 (Lewis [12]): Let $\nabla$ be an affine connection, and let $Y_{1}, \ldots, Y_{m}$ and $X_{1}, \ldots, X_{\bar{m}}$ be vector fields on a manifold $Q$. The control system in Equation $(5)$ is $(\mathcal{U}, \overline{\mathcal{U}})$ reducible to a system of the form in Equation (6) if and only if the following two conditions hold:

$$
\begin{aligned}
& \text { i ) } \operatorname{span}_{\mathbb{R}}\left\{X_{1}(q), \ldots, X_{m}(q)\right\} \\
& \operatorname{span}_{\mathbb{R}}\left\{Y_{1}(q), \ldots, Y_{\bar{m}}(q)\right\} \text { for each } q \in Q \text { (in } \\
& \text { particular, } \bar{m}=m) \\
& \text { ii })\langle X: Y\rangle \in \chi^{\infty}\left(D_{d y n}\right) \text { for every } X, Y \in \chi^{\infty}\left(D_{d y n}\right) \text {. }
\end{aligned}
$$

As noted in Lewis [12], the symmetric product plays a similar role in establishing $(\mathcal{U}, \widehat{U})$ reducibility to the Lie bracket in establishing controllability. The goal in the next section will be to extend Theorem 3.1 to the case of multiple model systems. It will turn out that such an extension is relatively straightforward and has natural interpretations in terms of the individual models making up the multiple model system. In particular, we will see that the only requirement on the multiple model system is that each model be $(\mathcal{U}, \overline{\mathcal{U}})$ reducible.

\section{Kinematic REDUCIBILITY FOR MUltiple MODEL SYSTEMS}

This section considers the problem of whether or not a multiple model system is kinematically reducible to an MMDA system. Let us start with a statement that should not be surprising. Lemma 4.1 states that if the solution to the dynamic equations only has switches which are separated by some small amount of time (making the switching signal piecewise continuous), the resulting solution is also kinematically reducible.

Lemma 4.1: Let $\Sigma$ be a multiple model system such that the switching signal $\sigma$ is piecewise constant. Then, $\Sigma$ is $(\mathcal{U}, \overline{\mathcal{U}})$ reducible iff $\Sigma_{\sigma_{i}, \cdots, \sigma_{j}}$ are all $(\mathcal{U}, \overline{\mathcal{U}})$ reducible.

Proof: Since $\sigma$ is piecewise constant, we know that the number of times that $\sigma$ changes are countable. Therefore, Iet the times when $\sigma$ changes its value be $\left\{t_{1}, t_{2}, \cdots,\right\}$ for $i$ in some index $I$. Then on the intervals $\left(t_{i}, t_{i+1}\right) \Sigma$ is $(\mathcal{U}, \overline{\mathcal{U}})$ reducible, making it $(\mathcal{U}, \overline{\mathcal{U}})$ reducible almost always. It therefore satisfies the requirements of Definition 3.6.

Note that Lemma 4.1 is already strong enough to guarantee reducibility for many systems. For instance, motion planning on stratified manifolds can be approached from a kinematic standpoint [10]. This approach can be validated by Lemma 4.1 for legged robots which are statically stable.

We will use Lemma 4.1 to prove Theorem 4.3, which says that solutions to the differential inclusion defined by multiple model systems are kinematically reducible if and only if the individual models are kinematically reducible. Before proving that this is true we will need the following simple result from Filippov [9].

Theorem 4.2 (Filippov [9]): Let $\mathbf{f}: Q \times \mathbb{R} \rightarrow T Q$ be a compact set-valued map and let $\left\{\Phi_{i}\right\}$ be a sequence of solutions to the differential inclusion

$$
\dot{q} \in \mathbf{f}(t, q)
$$


such that $\lim _{i \rightarrow \infty} \Phi_{i} \rightarrow \Phi$. Then $\Phi$ is also a solution to Equation (7).

We will use Theorem 4.2 several times in the proof of Theorem 4.3. Roughly speaking, we will use piecewise continuous $(\mathcal{U}, \overline{\mathcal{U}})$ reducible solutions of the multiple model mechanical system as approximations to arbitrary elements of $f$, and then use Theorem 4.2 to show that their kinematic counterparts on $T Q$ must also converge to an element of the differential inclusion defined on $T Q$.

Theorem 4.3: A multiple model system $\Sigma$ is $(\mathcal{U}, \overline{\mathcal{U}})$ reducible iff $\Sigma_{\sigma_{i}, \cdots, \sigma_{j}}$ are all $(\mathcal{U}, \overline{\mathcal{U}})$ reducible.

Proof: First note that it is obviously necessary that all the individual models be $(\mathcal{U}, \overline{\mathcal{U}})$ reducible in order for the resulting multiple model mechanical system to be reducible, because otherwise a perfectly valid solution to a multiple model mechanical system is the smooth, non-reducible solution. So let us show sufficiency. We must therefore show that when the individual models are $(\mathcal{U}, \overrightarrow{\mathcal{U}})$ reducible, the MMDA system satisfies parts $i$ ) and ii) of Definition 3.6.

(i) A multiple model mechanical system has the form

$$
{ }^{g} \nabla_{c^{\prime}(t)} c^{\prime}(t) \in u^{\alpha}{ }^{l} Y_{\alpha}(c(t))
$$

where $l \in \Lambda$ is the index for a given model, $g_{l}$ is the metric appropriate to that model, ${ }^{g_{l}} \nabla$ is the affine connection associated with $g_{l}$, and ${ }^{l} Y_{\alpha}^{i}$ is the $i^{\text {th }}$ component of vector field representing the force input corresponding to $u^{\alpha}$ of the $l^{\text {th }}$ model of the multiple model system. Equation (8) is equivalent to

$$
\ddot{q}^{i}+{ }^{g} \Gamma_{j k}^{i} \dot{q}^{j} \dot{q}^{k}=u^{\alpha l} Y_{\alpha}^{i} .
$$

Setting ${ }^{l} \mathcal{Y}_{\alpha}^{i}={ }^{g l} \Gamma_{j k}^{i} \dot{q}^{j} \dot{q}^{k}+u^{\alpha}{ }^{l} Y_{\alpha}^{i}$ and $Y_{\alpha}^{i}=\operatorname{co}\left\{{ }^{l} \mathcal{Y}_{\alpha}^{i}: l \in\right.$ $L\}$ we get that

$$
\ddot{q}^{i} \in \mathbf{Y}_{\alpha}^{i} .
$$

For a given solution $\Phi$ of Equation (10) we know that $\frac{d}{d t} \Phi \in \mathbf{Y}$, so we can choose a selection of $\mathbf{Y}$ which is locally representative of the time evolution. Denote this selection by $s(\mathcal{Y})_{\alpha}^{i} \in \mathbf{Y}$. Therefore,

$$
s(\mathbf{Y})_{\alpha}^{i}=\delta_{1} \mathcal{Y}_{\alpha_{1}}^{i}+\delta_{2} \mathcal{Y}_{\alpha_{2}}^{i}+\cdots+\delta_{m} \mathcal{Y}_{\alpha_{m}}^{i}
$$

such that $\delta_{j}>0$ and $\sum_{j}^{m} \delta_{j}=1$. Let us denote the composition of a flow $\Phi$ with itself $n$ times by $\Phi^{n}$. That is,

$$
\Phi^{n}(q)=\Phi \circ \Phi \circ \cdots \Phi \circ \Phi(q) .
$$

Dropping the index $i$, choose the following map to approximate the flow of the selection $s(\mathbf{Y})_{\alpha}^{i}$ :

$$
\Phi_{d y n}^{t, n}(q) \stackrel{\text { def }}{=}\left(\Phi^{\delta_{1} \mathcal{Y}_{\alpha_{1} \frac{t}{n}}} \circ \ldots \circ \Phi^{\delta_{m} \mathcal{Y}_{\alpha_{m}} \frac{t}{n}}\right)^{n}(q)
$$

$\Phi_{d y n}^{t, n}(q)$ consists of flows along $(\mathcal{U}, \overline{\mathcal{U}})$ reducible mechanical systems. Moreover, it is a solution of Equation (10) on $T Q$ which is absolutely continuous almost everywhere for every $n$. Lastly, it converges to the selection $s(\mathbf{Y})$ as $n \rightarrow \infty$. That is, by construction we get

$$
\lim _{n \rightarrow \infty} \Phi_{d y n}^{t, n}=\Phi^{s(\mathbf{Y})_{\alpha}}
$$

By assumption, we know that each segment $\Phi^{\delta_{i} \mathcal{Y}_{\alpha_{i}} \frac{t}{n}}$ of $\Phi_{d y n}^{t, n}$ is $(\mathcal{U}, \overline{\mathcal{U}})$-reducible. Therefore, for every choice of $n, \Phi_{d y n}^{t, n}$ is $(\mathcal{U}, \overrightarrow{\mathcal{U}})$ reducible by Lemma 4.1 . This then gives us, for each $n$, a corresponding map on $Q$ :

$$
\begin{aligned}
& \Phi_{\text {kin }}^{t, n}(q) \stackrel{\text { def }}{=} \tau_{Q} \circ \Phi_{d y n}^{t, n}(q)= \\
& \quad\left(\Phi^{\delta_{1} X_{a_{1}} \frac{t}{n}} \circ \Phi^{\delta_{2} X_{a_{2}} \frac{t}{n}} \cdots \cdots \Phi^{\delta_{m} X_{a_{m}} \frac{t}{n}}\right)^{n}(q)
\end{aligned}
$$

where each $\Phi^{\delta_{i} X_{a_{i}} \frac{t}{n}}$ is the reduced equations of $\Phi^{\delta_{i} \mathcal{Y}_{\alpha_{i}} \frac{t}{n}}$. Moreover, from Theorem 4.2 we know that $\lim _{n \rightarrow \infty} \Phi_{k i n}^{t, n}$ exists and that its limit is a solution to

$$
\dot{q} \in u^{a} \mathbf{X}_{a}
$$

where $\mathbf{X}_{a}=c o\left\{{ }^{l} X_{a} \mid l \in L\right\},{ }^{l} X_{a}$ are the reduced equations for a given model in Equation (6). Therefore, part i) of Definition 3.6 is satisfied.

(ii) This has the same essential steps as the above argument, but now we start with the kinematic solution and work towards a dynamic solution. Starting with the kinematic solutions from Equation (6), we know that for the model with index $l$ :

$$
\dot{q}^{i}=u^{a}{ }^{l} X_{a}^{i}
$$

Therefore, this MMDA system has kinematics of the form in Equation (15). Let us choose an arbitrary solution of Equation (15), and choose the selection $s\left(X_{a}\right)$ to be locally representative of the time evolution. That is, locally, we have $\Phi^{X_{a}}$, for $X_{a} \in \mathbf{X}_{\mathrm{a}}$. As before, we construct a sequence of solutions converging to $\Phi^{X_{a}}$. By construction, $\Phi_{k i n}^{t, n}$ satisfies this. We must show there exists a $\eta$ solution with

$$
\frac{d}{d t} \Phi^{X_{a}}=\eta
$$

We know that

$$
\lim _{n \rightarrow \infty} \Phi_{k i n}^{t, n}=\Phi^{X_{a}}\left(q_{0}, t\right)
$$

and that for every $n$ and $\Phi_{k i n}^{t, n}$ there exists a corresponding $\Phi_{d y n}^{t, n}$ (as defined in (i) above). Taking the limit of this, we have

$$
\lim _{n \rightarrow \infty} \Phi_{d y n}^{t, n}=\Phi^{Y_{\alpha}}
$$

which is a solution of Equation (10), again by Theorem 4.2. Taking the derivative of both sides, we get (after repeated application of the chain rule)

$$
\frac{d}{d t} \Phi^{Y_{\alpha}}=\frac{d}{d t} \lim _{n \rightarrow \infty} \Phi_{d y n}^{t, n}=\lim _{n \rightarrow \infty} \frac{d}{d t} \Phi_{d y n}^{t, n}=\lim _{n \rightarrow \infty} \Phi_{k i n}^{t, n}
$$

so part ii) is satisfied. 
Notice that the proof of Theorem 4.3 relied heavily on specifically constructing a solution with the desired properties based on known solutions to the individual models comprising the multiple model system.

This result shows that determining the kinematic properties of the individual models in a multiple model system is sufficient for determining the kinematic properties of the complete system. We should comment on the relationship between this result and controllability results which can be obtained for for multiple model systems. One of the intuitive aspects of Theorem 4.3 is precisely that it is sufficient for each model to be $(\mathcal{U}, \overline{\mathcal{U}})$ reducible in order to guarantee that the multiple model mechanical system is $(\mathcal{U}, \overline{\mathcal{U}})$ reducible. That is, piecewise $(\mathcal{U}, \overrightarrow{\mathcal{U}})$ reducibility is enough to guarantee $(\mathcal{U}, \overline{\mathcal{U}})$ reducibility across discontinuities. However, in the case of controllability, this no longer holds. We showed in a previous paper that an MMDA system can switch among individually controllable systems in such a way as to destroy controllability [19].

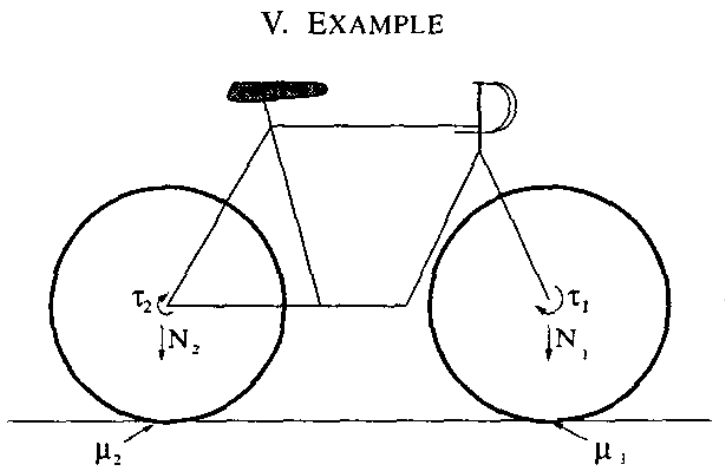

Fig. 1. Planar bicycle

As an example, consider a bicycle (Fig. 1) which is constrained to move on a line. The downward normal force on each wheel will depend on the bicycle's weight distribution. Assume that each wheel is actuated, with torques $\tau_{1}$ and $\tau_{2}$, and that each of the wheels may slip (depending upon the ground reaction force). Let $q=\left[x, \phi_{1}, \phi_{2}\right]^{T}$, where $\phi_{1}$ is the front wheel angle and $\phi_{2}$ is the rear wheel angle. Here $J$ is a wheel's moment of inertia about its rotational axis, $m$ is total bicycle mass, $R$ is the wheel radius, and $F_{i}^{R}=-\frac{\dot{x}-R \dot{\theta}_{i}}{\left\|\dot{x}-R \dot{\theta}_{i}\right\|} \mu_{i} N_{i}$. If $\lambda_{i}$ is the reaction force for each contact, the Coulomb friction model implies that the boundary between slipping and nonslipping states occurs at some value of $\lambda_{i}=\lambda_{n o m}$, thereby implying that the $\lambda$ space is divided into regions of different slipping states. Generally, for an $n$-contact system, the slipping regions are locally separated by hyperplanes which bound a hypercube. The problem of state determination arises from the inherently complicated dependency of $\lambda$ on the current state. The analysis based on Lagrangian mechanics suggests that there are four possible contact states, corresponding to neither wheel slipping, the front wheel slipping, the back wheel slipping, and both wheels slipping. We show that there exist a subset of solutions which are kinematic, in the sense defined earlier.

Now, using the mechanics as described in Section III, we see that the configuration space is $\left\{x, \phi_{1}, \phi_{2}\right\} \in \mathbb{R} \times S^{2}$, and the Riemannian metric describing the kinetic energy is

$$
g=(m+2 J) d x \otimes d x+J d \phi_{1} \otimes d \phi_{1}+J d \phi_{2} \otimes \phi_{2} .
$$

The two non-rolling constraints are

$$
\begin{aligned}
& \dot{x}-R \dot{\phi}_{1}=0 \\
& \dot{x}-R \dot{\phi}_{2}=0
\end{aligned}
$$

and the constraint covectors can be written as

$$
\begin{aligned}
& \omega_{1}=d x-R d \phi_{1} \\
& \omega_{2}=d x-R d \phi_{2}
\end{aligned}
$$

As inputs, we have

$$
F^{1}=d \phi_{1} \quad F^{2}=d \phi_{2} .
$$

Now, for each combination of slipping and no slipping, we have a set of equations to solve for. Therefore, we have four sets of equations to solve. Moreover, because the Christoffel symbols are all identically zero due to the simple form of the manifold and constraints, the equations depend entirely on the input forces and extemal forces due to friction.

\section{Case 1) No slipping}

In the case where there is no slipping, we can immediately see that the system must satisfy $\dot{\phi}_{1}=\dot{\phi}_{2}$. This, in turn, implies that the constraint distribution is

$$
R \frac{\partial}{\partial x}+\frac{\partial}{\partial \phi_{1}}+\frac{\partial}{\partial \phi_{2}} \text {. }
$$

Moreover, one can readily compute that the orthogonal complement of $D$ is

$$
-\frac{J}{m R} \frac{\partial}{\partial x}+\frac{\partial}{\partial \phi_{2}},-\frac{J}{m R} \frac{\partial}{\partial x}+\frac{\partial}{\partial \phi_{1}} .
$$

The associated input vector fields are

$$
Y_{1}=Y_{2}=\frac{1}{2 J+m R^{2}}\left(R \frac{\partial}{\partial x}+\frac{\partial}{\partial \phi_{1}}+\frac{\partial}{\partial \phi_{2}}\right)
$$

and the equations of motion are therefore:

$$
\ddot{q}=Y_{1} u^{1}+Y_{2} u^{2} .
$$

It is easy to see that $\left\langle Y_{1}, Y_{2}\right\rangle=0$, so this is a kinematic system.

\section{Case 2) and Case 3) One wheel slipping}

In the case where there is one wheel slipping, we may assume without loss of generality that the wheel is wheel number 1 . In this case, the constraint distribution is

$$
R \frac{\partial}{\partial x}+\frac{\partial}{\partial \phi_{1}}, \quad \frac{\partial}{\partial \phi_{2}} \text {. }
$$


Moreover, one can readily compute that the orthogonal complement of $D$ is

$$
-\frac{J}{m R} \frac{\partial}{\partial x}+\frac{\partial}{\partial \phi_{1}}
$$

Moreover, we can compute the reaction force due to the other wheel slipping. We should note that such a reaction force is still merely an external force, and will therefore just be added to the right hand side of Equation (5) with the associated $u^{a} \equiv 1$. If wheel 2 (the back wheel) is slipping, the reaction force is: $w_{2}\left(F_{2}^{R}\right)$. The associated input vector fields and external vector fields are

$$
\begin{aligned}
& Y_{1}=\frac{1}{2 J+m R^{2}}\left(R \frac{\partial}{\partial x}+\frac{\partial}{\partial \dot{\phi}_{1}}+\frac{\partial}{\partial \phi_{2}}\right) \\
& Y_{2}=\frac{1}{J} \frac{\partial}{\partial \phi_{2}} \\
& E=\frac{R^{2} F_{2}^{R}}{J+m R^{2}} \frac{\partial}{\partial x}+\frac{R F_{2}^{R}}{J+m R^{2}} \frac{\partial}{\partial \phi_{1}}-\frac{R F_{2}^{R}}{J} \frac{\partial}{\partial \phi_{2}}
\end{aligned}
$$

and the equations of motion are therefore:

$$
\ddot{q}=Y_{1} u^{1}+Y_{2} u^{2}+E .
$$

Now, to determine whether this system is kinematically reducible or not, we first note that $\left\langle Y_{1}, Y_{2}\right\rangle$ is again identically zero. Moreover, we should note that although Theorem 3.1 does not directly address the case of external forces, we can by direct inspection of Definition 3.6 see that if $E \notin$ $\operatorname{span}\left\{Y_{i}\right\}$ then the system cannot in general be reducible. However, if $E \in \operatorname{span}\left\{Y_{i}\right\}$ and the $\left\{Y_{i}\right\}$ satisfy the conditions for reducibility, then the system is automatically reducible because the external forces are "covered" by the inputs. Therefore, we need only check that $E$ lies in the span of $Y_{1}$ and $Y_{2}$. Moreover, it can be easily computed that $E \in \operatorname{span}\left\{Y_{1}, Y_{2}\right\}$. Therefore, this system is kinematically reducible. Note that this property does not depend on the particular description of the reaction force, and is moreover invariant with respect to the reaction forces differentiability.

\section{Case 4) Both wheels slipping}

In the case where both wheels are slipping, there are no constraints to enforce. In this case, the constraint distribution does is identically zero and the orthogonal complement is trivially the entire tangent space. Moreover, we can compute the reaction force due to the wheels slipping to be $w_{1}\left(F_{1}^{R}\right)$ and $w_{2}\left(F_{2}^{R}\right)$. The associated input vector fields and external vector fields are

$$
\begin{aligned}
& Y_{1}=\frac{1}{J} \frac{\partial}{\partial \phi_{1}} \\
& Y_{2}=\frac{1}{J} \frac{\partial}{\partial \phi_{2}} \\
& E=\frac{F_{2}^{R}+F_{1}^{R}}{m} \frac{\partial}{\partial x}-\frac{R F_{1}^{R}}{J^{2}} \frac{\partial}{\partial \phi_{1}}-\frac{R F_{2}^{R}}{J^{2}} \frac{\partial}{\partial \phi_{2}}
\end{aligned}
$$

and the equations of motion are therefore:

$$
\ddot{q}=Y_{1} u^{1}+Y_{2} u^{2}+E .
$$

In this case, it is clear that $E \notin \operatorname{span}\left\{Y_{1}, Y_{2}\right\}$. Therefore this system (not surprisingly) is not kinematically reducible. However, there may be a more general sense in which this system or another system satisfying $E \notin \operatorname{span}\left\{Y_{1}, Y_{2}\right\}$ could be thought of as kinematically reducible. In particular, if $\left\langle E, Y_{i}\right\rangle=0 \forall i$, then one can imagine that this system could be reduced to a kinematic system with drift. However, a treatment such as the one presented here cannot be applied without caution-in general, friction models are not differentiable everywhere, although they are typically differentiable away from the stick/slip point (see [21]). This is one focus of future research.

\section{SOME FinAL REMARKS}

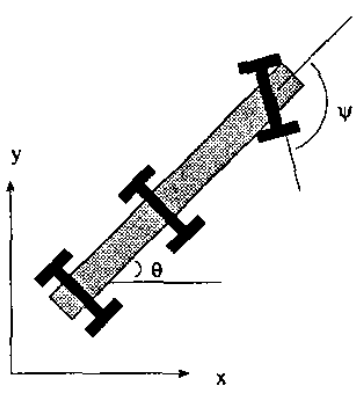

Fig. 2. Simplified Mars Rover

This paper discusses and derives conditions for kinematic reducibility of multiple model systems. This structure is put to advantage in [18] in an application to distributed manipulation and in [19] where we analyze the controllability properties of an example like that found in Figure 2. Kinematic reducibility can be related to the power dissipation method, a method for determining the quasistatic equations of motion for an overconstrained system (see [2], [23]). We have been able to show that the solutions to the power dissipation method correspond to kinematic solutions of multiple model systems. For details, see the expanded version of this paper [20].

We finish with a comment on the interesting example in Figure 2 inspired by the Mars rover. This has three wheels, with all three wheels driven. We can consider its configuration space to be $\left(x, y, \theta, \psi, \phi_{1}, \phi_{2}, \phi_{3}\right)$. This system has six nonholonomic constraints (one associated with each wheel having both a no roll constraint and a no sideways slip constraint). Therefore, there are $2^{6}=64$ possible models governing the dynamics of the vehicle. For this reason, we do not relate all the calculations for this vehicle. However, it is easy to show, using a symbolic mathematics package such as Mathematica, that this system also has a subset of kinematic solutions, and that these solutions correspond to the solutions of the power dissipation method for this system. Such a correspondence is important because the power dissipation method is very straight forward to solve and these solutions can be used for both controllability analysis and for purposes of motion planning (see [17], [19]). 


\section{REFERENCES}

[1] R. Abraham, J.E. Marsden, and T.S. Ratiu. Manifolds, Tensor Analysis, and Applications. Addison-Wesley, 1988.

[2] J.C. Alexander and J.H. Maddocks. On the kinematics of wheeled vehicles. The International Joumal of Robotics Research, 8(5):15-27, October 1989.

[3] E. Asarin, O. Bournez, T. Dang, O. Maler, and A. Pneuli. Effective synthesis of switching controllers for linear systems. Proc. IEEE, 88(7):1011-1025, July 2000. Special Issue "Hybrid Systems: Theory and Applications".

[4] A.M. Bloch, P. S. Krishnaprasad, J.E. Marsden, and R.M. Murray. Nonholonomic mechanical systems with symmetry. Arch. Rational Mech. Anal., 136(1):21-99, 1996.

[5] M.S. Branicky. Multiple Lyapunov functions and other analysis tools for switched and hybrid systems. IEEE Trans. Automatic Control, 43(4):475-482, April 1998.

[6] F. Bullo, A.D. Lewis, and K.M. Lynch. Controllable kinematic reductions for mechanical systems: Concepts, computational tools, and examples. In International Symposium on Mathematical Theory of Networks and Systems (MTNS), 2002.

[7] F. Bullo and K.M. Lynch. Kinematic controllability for decoupled trajectory planning in underactuated mechanical systems. IEEE Transactions on Robotics and Automation, 17(4):402-412, August 2001.

[8] W.P. Dayawansa and C.F. Martin. A converse Lyapunov theorem for a class of dynamical systems which undergo switching. IEEE Trans. Automatic Control, 44(4):751-760, Apr. 1999.

[9] A.F. Filippov. Differential Equations with Discontinuous Right-Hand Sides. Kluwer, 1988.

[10] B. Goodwine and J.W. Burdick. Controllability of kinematic control systems on stratified configuration spaces. IEEE Trans. on Automatic Control, 46(3):358$368,2000$.

[11] J.P. Hespanha, D. Liberzon, and A.S. Morse. Logicbased switching control of a nonholonomic system with parametric uncertainty. Systems Control Lett., 38:167177, 1999.

[12] A.D. Lewis. When is a mechanical control system kinematic? In Proc. $38^{\text {th }}$ IEEE Conf. on Decision and Control, pages 1162-1167, Dec. 1999.

[13] A.D. Lewis. Simple mechanical control systems with constraints. IEEE Transactions on Automatic Control, 45(8):1420-1436, 2000.

[14] D. Liberzon and A.S. Morse. Basic problems in stability and design of switched systems. IEEE Control System Mag., 19(5):59-70, 1999.

[15] J.E. Marsden and J.P. Ostrowski. Symmetries in motion:
Geometric foundations of motion control. Nonlinear Science Today, 1998.

[16] J.E. Marsden and T.S. Ratiu. An Introduction to Mechanics and Symmetry. Springer-Verlag, 1992.

[17] T. D. Murphey and J. W. Burdick. A controllability test and motion planning primitives for overconstrained vehicles. In Proc. IEEE Int. Conf. on Robotics and Automation, Seoul, Korea, 2001.

[18] T. D. Murphey and J. W. Burdick. Global stability for distributed systems with changing contact states. In Proc. IEEE Int. Conf. on Intelligent Robots and Systems, Hawaii, 2001.

[19] T. D. Murphey and J. W. Burdick. Nonsmooth controllability and an example. In Proc. IEEE Conf. on Decision and Control (CDC), Washington D.C., 2002.

[20] T.D. Murphey and J.W. Burdick. The power dissipation method and kinematic reducibility for multiple model systems. IEEE Transactions on Robotics and Automation. To Be Submitted.

[21] H. Olsson, K.J. Astrom, C. Canudas de Wit, M. Gafvert, and $\mathrm{P}$. Lischinsky. Friction models and friction compensation. European Journal of Control, Preprint.

[22] G.J. Pappas, G. Laffierier, and S. Sastry. Hierarchically consistent control sytems. IEEE Trans. Automatic Control, 45(6):1144-1160, June 2000.

[23] M. A. Peshkin and A. C. Sanderson. Minimization of energy in quasistatic manipulation. IEEE Transactions on Robotics and Automation, 5(1), February 1989.

[24] M. Zefran and J.W. Burdick. Design of switching controllers for systems with changing dynamics. Proc. Conf. on Decision and Control, 1998. 